\title{
Fabrication and characterization of conductive microcapsule containing phase change material
}

https://doi.org/10.1515/epoly-2019-0055

Received June 22, 2019; accepted August 04, 2019.

\begin{abstract}
Microencapsulated phase change materials (MicroPCMs) were fabricated using $n$-octadecane as PCM and melamine-formaldehyde as shell via in situ polymerization. They were coated with polypyrrole (PPy) to fabricate conductive microcapsules. The structure, morphology, thermal properties and the electrical conductivity of the microcapsules were characterized using the scanning electron microscope (SEM), the Fourier transformed infrared spectroscopy (FTIR), the thermo gravimetric analysis (TGA), the differential scanning calorimetry (DSC) and the standard four-probe method. The results show that, $n$-octadecane is well encapsulated in rough and compact spherical composites. The melting and freezing the composites latent heats are 90.2 and $92.0 \mathrm{~J} / \mathrm{g}$, respectively, while the mass percentage of the $n$-octadecane in the composites is $49.7 \%$. The melting and crystallizing peak temperature of PPy/MicroPCMs is $24.6^{\circ} \mathrm{C}$ and $17.9^{\circ} \mathrm{C}$, respectively. The addition of PPy improves the thermal stability of the composites. The conductivity of the PPy/MicroPCMs increases from $0.1 \mathrm{~S} \cdot \mathrm{cm}^{-1}$ to $0.33 \mathrm{~S} \cdot \mathrm{cm}^{-1}$ as the PPy concentration increases from 3 to $10 \mathrm{wt} \%$.
\end{abstract}

Keywords: thermal energy storage; MicroPCMs; polypyrrole; electrical conductivity

\section{Introduction}

The energy shortage problem has gained great importance in recent years. Thermal energy storage is a favorable way

\footnotetext{
* Corresponding author: Xing-Xiang Zhang, School of Material Science and Engineering, Tianjin Polytechnic University, Tianjin 300387, China, email: zhangpolyu@aliyun.com; Tel/Fax: +86 22 83955054

Hao-Ran Yun, Chun-Lei Li, School of Material Science and Engineering, Tianjin Polytechnic University, Tianjin 300387, China.
}

to improve efficient energy management and utilization. One of favored methods to store thermal energy is latent heat storage, because of its high storage density and small temperature variation from storage to retrieval (1). Phase change materials (PCMs) can absorb and release large capacity of latent energy during the phase change process when the ambient temperature is changed within a certain range (2). Thence, PCMs have a wide application prospect in the field of energy conservation, such as energy efficient buildings, solar energy storage, and thermo-regulated fibers (3-10).

The most widely applied organic solid-liquid PCMs are paraffin, polyether, polyester, and fatty acids. A container was used to prevent leakage of melted PCMs; however, it could result in extra thermal resistance and cost (11). Microencapsulated phase change materials (MicroPCMs), which are tiny particles with core materials of PCMs surrounded with organic or inorganic materials, are used for many applications. Straight-chain paraffin and fatty acids are widely used as core materials due to their different phase-transition temperatures, and the most often used shell materials are polymers. Microcapsules and nanocapsules were prepared by in situ polymerization, in which $n$-alkanes (n-octadecane, $n$-nonadecane, and $n$-eicosane) were used as core materials with melamine-formaldehyde, poly(vinyl alcohol), urea-melamine-formaldehyde and polyurethane as shells (12-17). Also, poly(methyl methacrylate) (18), expanded perlite composite (19), silica fume composite (20) and cellulose acetate (21) were selected as shell materials for thermal energy storage. Furthermore, polyaniline/phase-change nanocapsule composite, which is infrared sensitive, was synthesized by in situ polymerization (22).

However, there is still a little information available for electric conductive MicroPCMs. In recent years, conductive polymer composites have become a new hot spot in the study of conductive composite materials. Not only can composite materials with conductive properties be prepared, but also some new properties such as light, magnetism, antistatic and anticorrosion can be imparted, so this will expand application of 
MicroPCMs. The composite material prepared by in-situ polymerization method can make the conductive layer and the matrix better composite, and obtain a conductive composite material with regular morphology and stable performance.

In the present work, we fabricated and investigated the properties of a core-shell microcapsule of $\mathrm{PPy} / n$-octadecane via in situ chemical oxidative polymerization of pyrrole. PPy has high thermal stability and electrochromic (23). Yang et al. prepared carbon/ polycarbazole nanocomposite black powder by in-situ polymerization at $0^{\circ} \mathrm{C}$ with ammonium persulfate as oxidant (24). Chao et al. used in-situ polymerization method with APS as the initiator and poly(2-hydroxy-3(methacryloyloxy) propane-1-sulfonate) as surfactant and dopant on the surface of $\mathrm{CB}$ nanoparticles. The $\mathrm{CB} / \mathrm{PPy}$ nanocomposite had a core-shell structure and the thickness of polypyrrole layer was from 30 to $100 \mathrm{~nm}$ (25). However, there are few studies on the preparation of conductive polypyrrole phase change composites with core-shell structure by in-situ polymerization. So we expect the PCM microcapsules coated with PPy can exhibit electrical conductivity and phase change properties, which will be possible to widen the application of PCMs in camouflage technology and electronic cooling. The results show that PPy/MicroPCMs have higher latent heat and the conductivity of the PPy/MicroPCMs increases from $0.1 \mathrm{~S} \cdot \mathrm{cm}^{-1}$ to $0.33 \mathrm{~S} \cdot \mathrm{cm}^{-1}$ as the PPy concentration increases from 3 to $10 \mathrm{wt} \%$ and it reached the design goals.

\section{Experimental}

\subsection{Materials}

Pyrrole (Py) monomer (98 wt\%, Sigma-Aldrich) was used after vacuum distillation. $\mathrm{N}$-octadecane (purity 99 wt\%, Union Lab Supplies, Hong Kong) was used as core material. Melamine (98 wt $\%$, Tianjin Resins Material Factory) and formaldehyde (37 wt $\%$ aqueous, A.R., Tianjin Chem. Co.) were used as monomers. Anionic surfactant, SMA (sodium salt of styrenemaleic anhydride copolymer, $19 \mathrm{wt} \%$ aqueous solution, Shanghai Leather Chemical Works) was employed as an emulsifier. Acetic acid (36 wt\%), triethanolamine (95 wt\%), and sodium hydroxide (A.R.), as $\mathrm{pH}$ regulators, were from Tianjin Chemical Reagents. $\mathrm{CuCl}_{2} \cdot 2 \mathrm{H}_{2} \mathrm{O}$ (99 wt\%, Guangfu Fine Chemical Research Institute, Tianjin) was used as oxidant.

\subsection{Methods}

\subsubsection{Fabrication of MicroPCMs}

In $150 \mathrm{~mL}$ of distilled water, five and one fifth gram of SMA was dispersed, and heated to $60^{\circ} \mathrm{C} . N$-octadecane was added to the micelles, the mixture was emulsified mechanically by the homomixer at a stirring rate of $3200 \mathrm{rpm}$ for $30 \mathrm{~min}$. The prepolymer was synthesized in a round bottomed flask equipped with a mechanical stirrer. Afterward, $13 \mathrm{~mL}$ formaldehyde, $7 \mathrm{~g}$ melamine, and $7 \mathrm{~mL}$ distilled water were added to the flask. The $\mathrm{pH}$ was adjusted to approximately 8-9 with triethanolamine. Then, $3 \mathrm{~mL}$ pyrrole was added into the mixture under vigorous stirring at $70^{\circ} \mathrm{C}$ in a nitrogen atmosphere until the mixture became transparent. After the $\mathrm{pH}$ of the emulsion was regulated to about 4-5, prepolymers were dropped at the rate of nearly $1 \mathrm{~mL} / \mathrm{min}$ to the emulsion under vigorous stirring at $60^{\circ} \mathrm{C}$ for $2.5 \mathrm{~h}$. After the reaction completed, MicroPCMs were obtained.

\subsubsection{Fabrication of PPy/MicroPCMs}

The composite of PPy/MicroPCMs was prepared by oxidative polymerization. A mixture of $\mathrm{Py}$ and $\mathrm{CuCl}_{2}$ was prepared. First, $3 \mathrm{~mL}$ pyrrole was added into the prepolymer. The molar ratio of $\mathrm{Py} / \mathrm{CuCl}_{2}$ was 1:1 (26). Then, an aqueous solution $(50 \mathrm{~mL})$ of $\mathrm{CuCl}_{2} \cdot 2 \mathrm{H}_{2} \mathrm{O}$ was added dropwise to the mixture. The mixture was mechanical stirring for $6 \mathrm{~h}$ under $60^{\circ} \mathrm{C}$ and the rotating speed should be $300 \mathrm{r} / \mathrm{min}$. Then, the precipitates were filtered and washed with deionized water and ethanol. And were vacuum dried at $40^{\circ} \mathrm{C}$ for $24 \mathrm{~h}$.

\subsubsection{Fabrication of PPy}

The pure PPy was prepared by oxidative polymerization with $3 \mathrm{~mL}$ pyrrole and an aqueous solution $(50 \mathrm{~mL})$ of $\mathrm{CuCl}_{2} \cdot 2 \mathrm{H}_{2} \mathrm{O}$ for $6 \mathrm{~h}$ at $60^{\circ} \mathrm{C}$. Then, the precipitates were filtered, washed with deionized water and ethanol, and were vacuum dried at $40^{\circ} \mathrm{C}$ for $24 \mathrm{~h}$ (Table 1 ).

\subsection{Characterization}

A drop of the colloidal solution was extracted and spread on a cover glass. The surface morphologies of vacuum dried MicroPCMs at $40^{\circ} \mathrm{C}$ for $24 \mathrm{~h}$ were examined using a field emission scanning electron microscope (FESEM, S-4800, Hitachi Co., Japan). 
Table 1: Conditions for preparation of experimental samples.

\begin{tabular}{lcccc}
\hline Sample & Pyrrole $(\mathrm{mL})$ & $\begin{array}{c}\text { The mass fraction of pyrrole } \\
\text { in the prepolymer }(\%)\end{array}$ & Reaction temperature $\left({ }^{\circ} \mathrm{C}\right)$ & Reaction time $(\mathrm{h})$ \\
\hline S-1 & 1.5 & - & 60 & 12 \\
S-2 & 0.9 & 3 & 60 & 12 \\
S-3 & 1.5 & 5 & 60 & 12 \\
S-4 & 2.1 & 7 & 60 & 12 \\
S-5 & 3.0 & 10 & 60 & 12 \\
S-6 & 1.5 & 5 & 40 & 12 \\
S-7 & 1.5 & 5 & 50 & 12 \\
S-8 & 1.5 & 5 & 70 & 12 \\
\hline
\end{tabular}

FTIR spectra of $n$-octadecane, composite shells and MicroPCMs were determined using a Fourier transformed infrared spectroscopy (FTIR, TENSOR37, Bruker Co., Germany) at room temperature.

Thermal properties were measured using differential scanning calorimetry (DSC, PERKIN-ELMER DSC-7) in the range of $0-80^{\circ} \mathrm{C}$ at a heating or cooling rate of $10^{\circ} \mathrm{C} / \mathrm{min}$ in a nitrogen atmosphere. The content of n-octadecane was calculated (Eq. 1):

$$
\eta \%=\Delta \mathrm{H}_{\mathrm{MEPCM}} / \Delta \mathrm{H}_{\mathrm{PCM}} \times 100
$$

where, $\eta$ - the mass percentage of the PCM in the composites; $\triangle H_{\text {MEPCM }}$ - the melting latent heat of the composites, and $\Delta H_{P C M}$ - the melting latent heat of the $n$-octadecane as measured by the DSC.

Thermal stability of MicroPCMs and n-octadecane was examined by thermo gravimetric analysis (TGA, 409 $\mathrm{PC} / \mathrm{PG}$, Netzsch Co., Germany) in the range of $25-800^{\circ} \mathrm{C}$ at a heating rate of $10^{\circ} \mathrm{C} / \mathrm{min}$, in a nitrogen atmosphere.

The conductivity of MicroPCMs at room temperature was measured by a standard four-probe (Four-probe Technology Co., Guangzhou) method on pressed pellets of composites under $20 \mathrm{MPa}$.

\section{Results and discussion}

\subsection{Morphology and structure}

Figure 1 shows the SEM micrographs of PPy/MicroPCMs. It can be seen that most composites are spherical and have a rough surface. PPy is located on the shell of the PPy/MicroPCMs. The diameter of the PPy/MicroPCMs is approximately 20-30 $\mu \mathrm{m}$. In addition, when the content of polypyrrole is $3 \mathrm{wt} \%(\mathrm{~s}-2)$, the surface of microcapsules cannot form a continuous distribution. When the content of polypyrrole is $10 \mathrm{wt} \%$ (s-5), the content of polypyrrole on the surface of microcapsules is high, and the surface aggregation is high. It is smooth.

As shown in Figure 2, the inner surface of $\mathrm{PPy} /$ MicroPCMs is extremely smooth. When the content of pyrrole monomer is $5 \mathrm{wt} \%(\mathrm{~s}-3)$, the outer surface of the polypyrrole composite microcapsules prepared is compact, smooth and uniform in thickness.

As shown in Figure 3, when the reaction temperature is $40^{\circ} \mathrm{C}$, it is hard to prepare the composite into a sphere, and there are many flaky impurities (s-6). When the reaction temperature is $50^{\circ} \mathrm{C}$, the microspheres surface is smooth, the polypyrrole is less attached, and a large amount of smooth flaky material exists. When the reaction temperature is $60^{\circ} \mathrm{C}(\mathrm{s}-3)$ and $70^{\circ} \mathrm{C}(\mathrm{s}-8)$, a core-shell composite with polypyrrole on the surface can be formed, but the composite at $70^{\circ} \mathrm{C}$ is not a regular spherical shape and the surface layer is aggregated. The thickness of the pyrrole is not uniform.

It can be seen from the above analysis that when the reaction temperature is $60^{\circ} \mathrm{C}$ and the content of pyrrole monomer is $5 \mathrm{wt} \%(\mathrm{~s}-3)$ and $7 \mathrm{wt} \%(\mathrm{~s}-4)$, the prepared polypyrrole microcapsules are spherical in shape and the thickness of the surface polypyrrole is uniform.

The diameter of the PPy/MicroPCMs is approximately 20-30 $\mu \mathrm{m}$. The inner surface of PPy/MicroPCMs is extremely smooth. (s-3) PPy/MicroPCMs tend to be dispersed without agglomeration. However, PPy does not fully coat the melamine resin layer and part of the Py monomer self-polymerized.

\subsection{Thermal properties}

The FT-IR spectra of $n$-octadecane, PPy, MicroPCMs, PPy/MicroPCMs are shown in Figure 4. The main characteristic absorption peaks of n-octadecane 


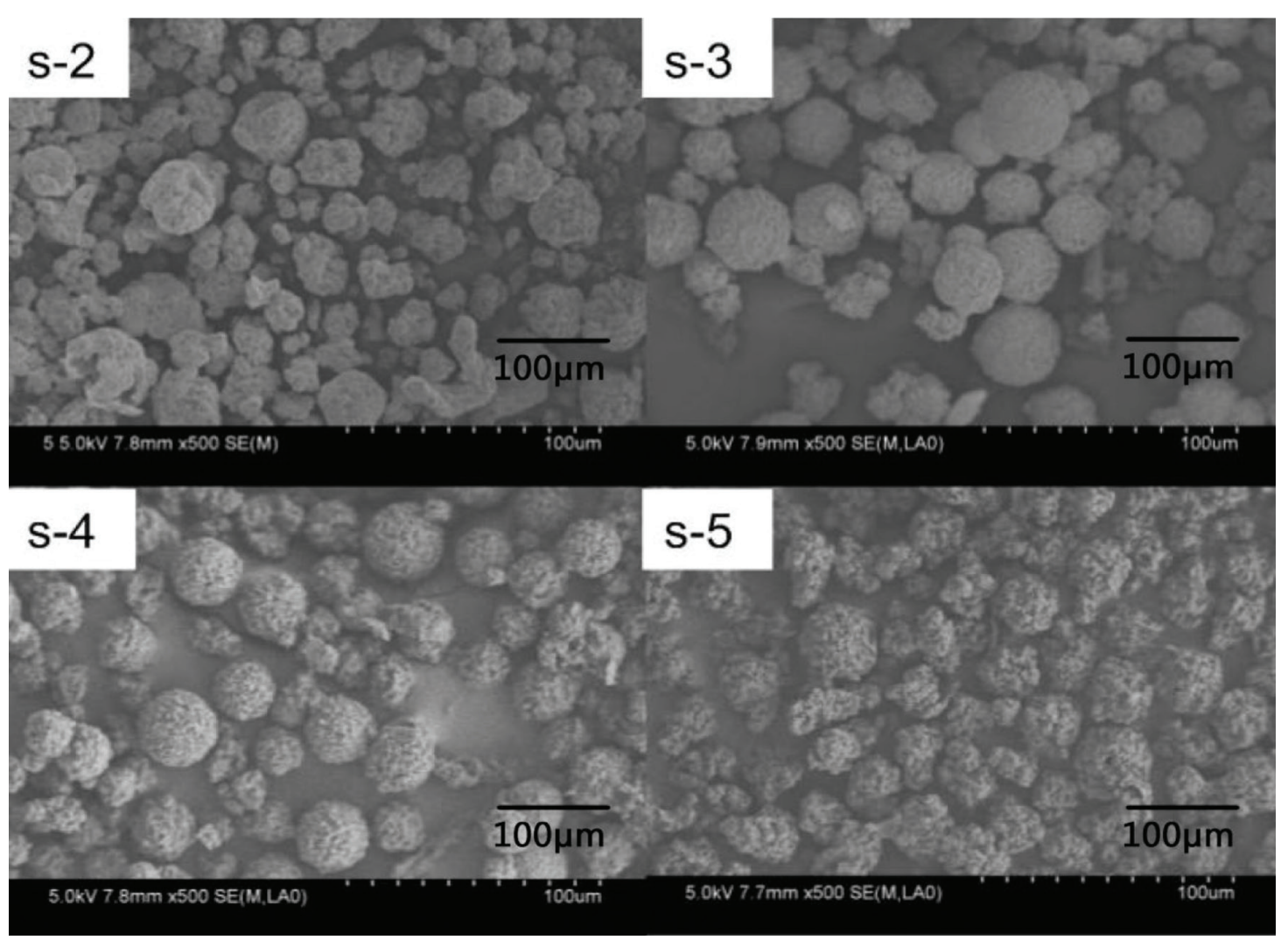

Figure 1: SEM of polypyrrole and polypyrrole monomer with different pyrrole monomer content.

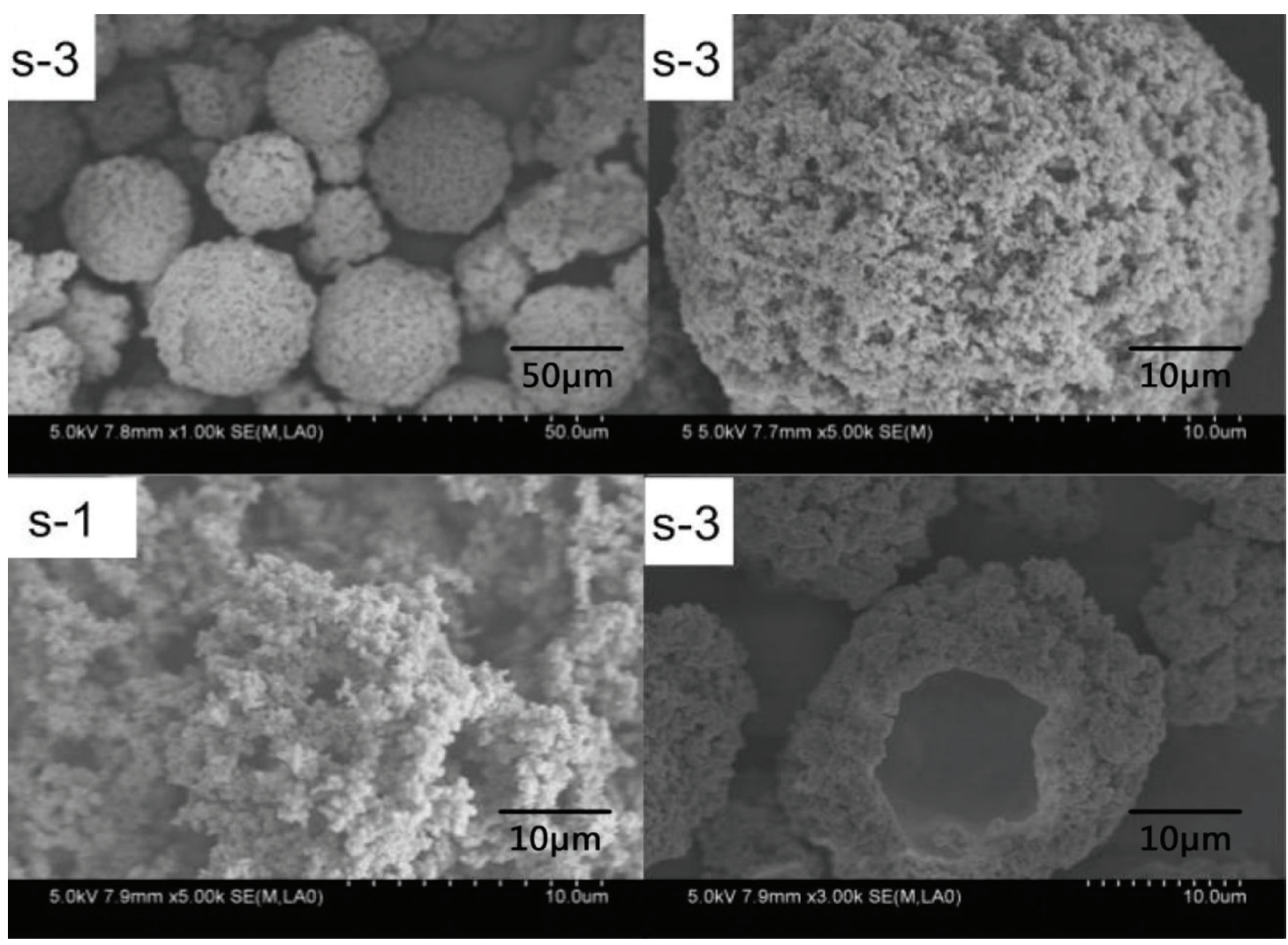

Figure 2: Enlarged picture of different multiples of composites prepared with the pyrrole monomer content of $5 \%$. 

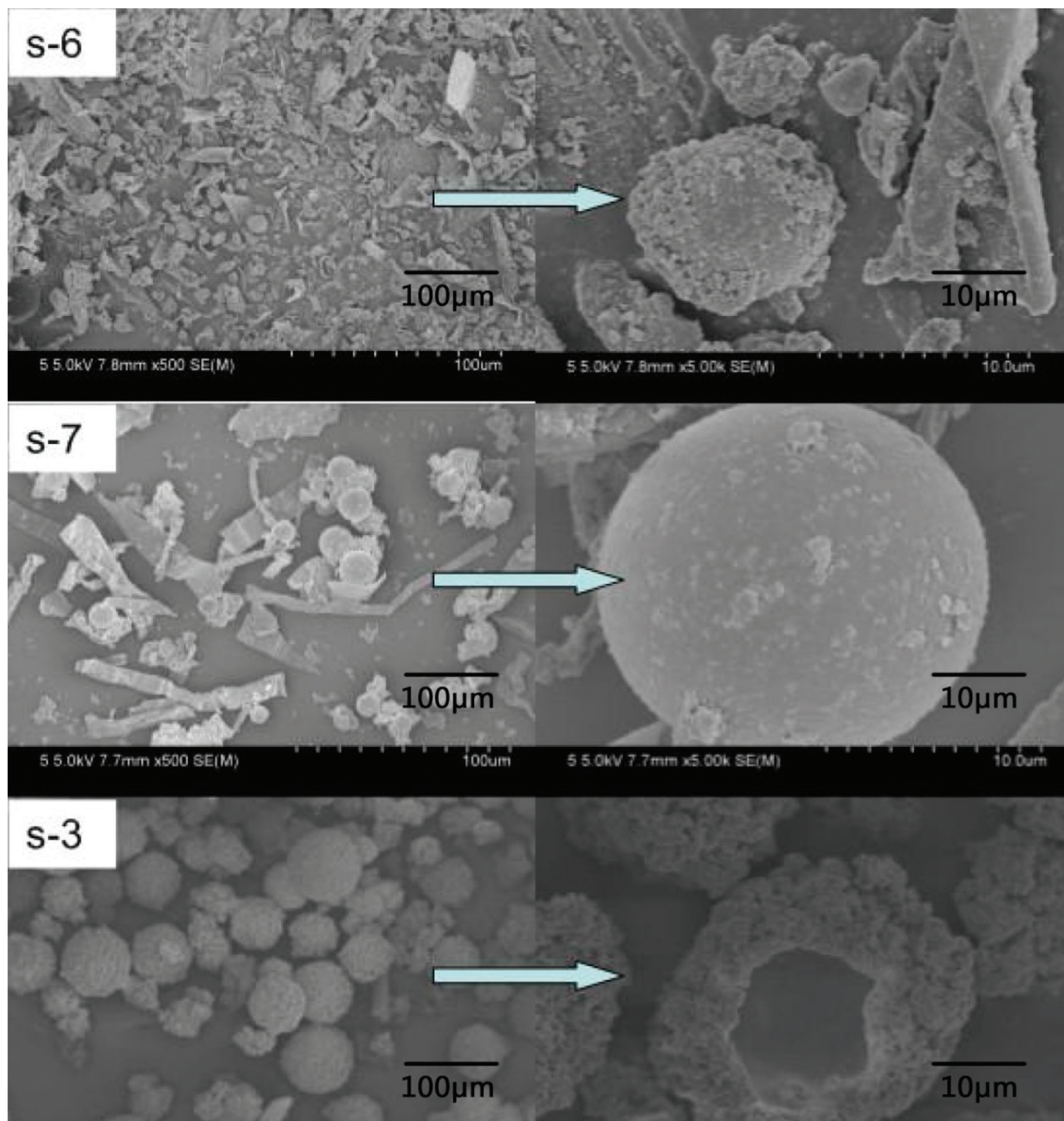

$5.0 \mathrm{kV} 7.9 \mathrm{~mm} \times 500$ SEE (M,LAD)

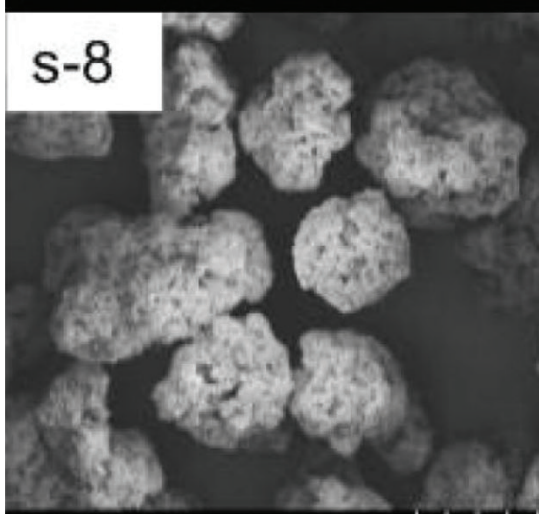

55 OKV 7 Bmm x500 SEIM?

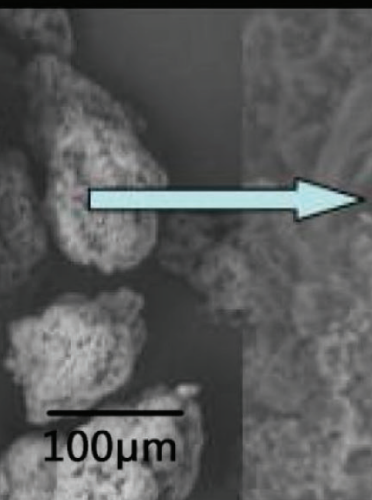

icón'

Figure 3: SEM image of polypyrrole composite prepared at different reaction temperatures. 


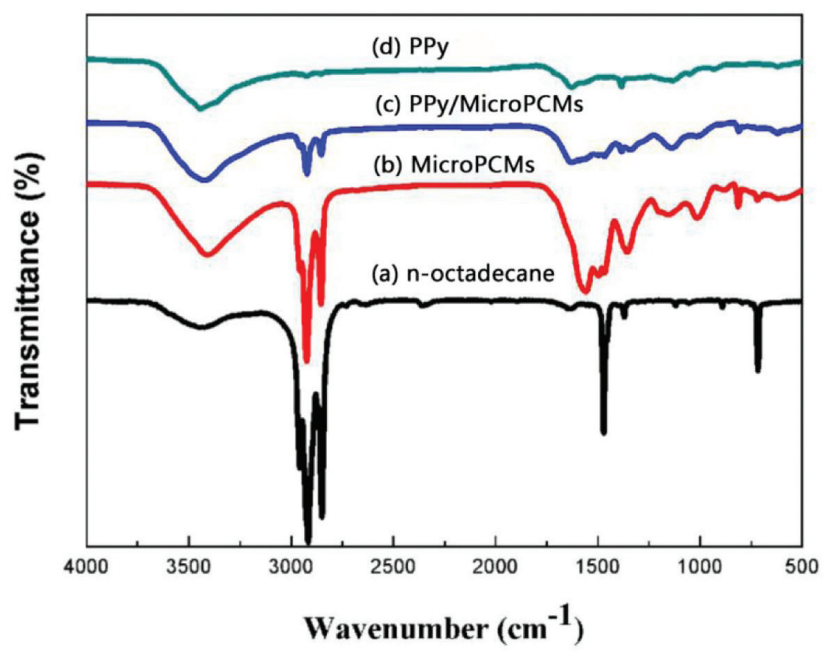

Figure 4: FT-IR spectra of (a) $n$-octadecane, (b) MicroPCMs, (c) PPy/MicroPCMs, (d) PPy.

at $2857 \mathrm{~cm}^{-1}, 2923 \mathrm{~cm}^{-1}$ and $2853 \mathrm{~cm}^{-1}$ are due to the aliphatic $\mathrm{C}-\mathrm{H}$ stretching vibration. The peaks corresponding to $\mathrm{C}-\mathrm{H}$ stretching vibration of composite shells are overlapped with $n$-octadecane in each spectrum of MicroPCMs (Figures $4 \mathrm{~b}$ and $4 \mathrm{c}$ ). The peaks at around $1470 \mathrm{~cm}^{-1}, 1356 \mathrm{~cm}^{-1}$ and $720 \mathrm{~cm}^{-1}$ are assigned to the $\mathrm{C}-\mathrm{H}$ bending vibration, which can be observed in the spectra of $n$-octadecane, MicroPCMs and PPy/MicroPCMs, and are the characteristic bands for $n$-octadecane (15). As shown in Figure $4 \mathrm{~d}$, the absorption peak at $3400 \mathrm{~cm}^{-1}$ is related to the $\mathrm{N}-\mathrm{H}$ stretching vibration of pyrrole rings; and $1633 \mathrm{~cm}^{-1}$ is assigned to the stretching vibration of the conjugated $\mathrm{C}=\mathrm{C}$ double bond of pyrrole rings; and $1400 \mathrm{~cm}^{-1}$ is attributed to the $\mathrm{C}-\mathrm{N}$ stretching vibration, while $620 \mathrm{~cm}^{-1}$ is assigned to $\mathrm{N}-\mathrm{H}$ out of plane vibration. The spectra of the PPy/MicroPCMs include all the characteristic peaks of PPy and $n$-octadecane; and the spectra of the MicroPCMs do not include the characteristic peaks of PPy. The characteristic peaks of PPy and $n$-octadecane are stronger than those of the MicroPCMs.

DSC heating and cooling curves of $n$-octadecane, MicroPCMs and PPy/MicroPCMs are shown in Figure 5. The data are listed in Table 2. It is obviously seen that the temperatures of MicroPCMs and PPy/MicroPCMs are very close to those of the $n$-octadecane. The melting and crystallizing peak temperature of PPy/MicroPCMs is $24.6^{\circ} \mathrm{C}$ and $17.9^{\circ} \mathrm{C}$, respectively. The encapsulation ratio can be calculated with Eq. 1. The encapsulation ratio of MicroPCMs and PPy/MicroPCMs is $40.1 \%$ and $40.4 \%$, respectively. The encapsulation ratio of MicroPCMs is higher than that of PPy/MicroPCMs, and could be attributed to two points: (1) the PPy has good thermal conductivity; (2) due to the fact

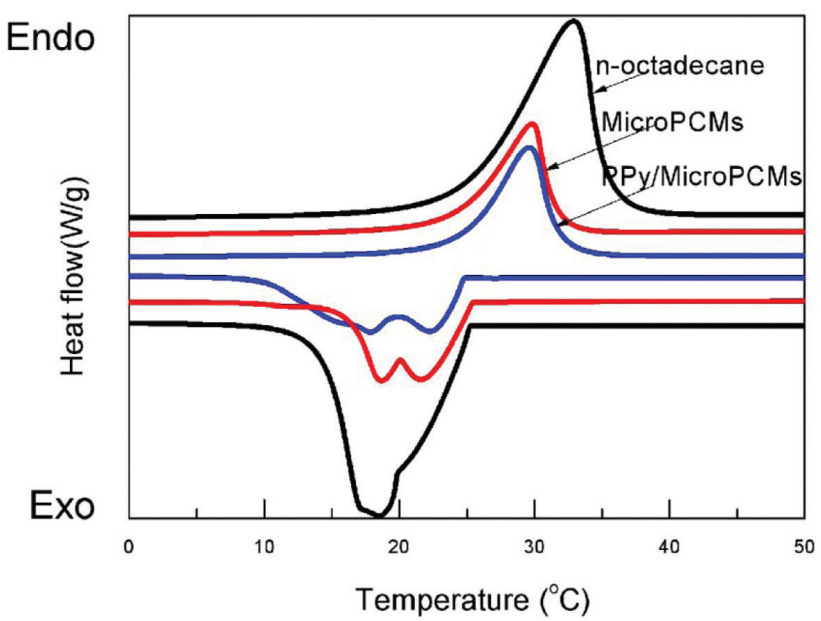

Figure 5: DSC curves of $n$-octadecane, MicroPCMs and PPy/MicroPCMs.

that not entire $n$-octadecane is encapsulated in MicroPCMs, the pores which are formed in the polymerization process of the pyrrole monomer provide many places for some free $n$-octadecane.

Figure 6 shows the TGA curves of $n$-octadecane, MicroPCMs, PPy/MicroPCMs and PPy. The mass of all composites decreases with increasing temperature. The $n$-octadecane starts to lose mass at nearly $160^{\circ} \mathrm{C}$ and decomposes completely at approximately $200^{\circ} \mathrm{C}$. The melamine resin shell protects $n$-octadecane from losing mass quickly, and the leakage of $n$-octadecane finishes at about $250^{\circ} \mathrm{C}$. The temperature range of $250-430^{\circ} \mathrm{C}$ is caused by the further decomposition of melamine resin. The improvement of thermal stability of PPy/MicroPCMs is obviously seen in Figure 4b. The first mass loss is due to $n$-octadecane leaking out of the shell materials, while the second mass loss starts at about $250^{\circ} \mathrm{C}$. This could result from the decomposition of the degradation of melamine resin. The third mass loss starts at about $418^{\circ} \mathrm{C}$, mainly due to the PPy chains. Owing to the PPy content in $\mathrm{PPy} / \mathrm{MicroPCMs}$, the mass loss rate of MicroPCMs is higher than that of PPy/MicroPCMs. This indicates that PPy improves the thermal stability of the composites due to the higher thermal stability of the PPy itself, and the synergistic effect between the PPy and melamine resin.

\subsection{Electrical conductivity of PPy/MicroPCMs}

Effects of PPy contents in fabrication of PPy/MicroPCMs on conductivity are shown in Figure 7. The room conductivity of PPy/MicroPCMs is significantly affected by the contents 
Table 2: Thermal property of $n$-octadecane and PPy/MicroPCMs.

\begin{tabular}{|c|c|c|c|c|c|c|}
\hline \multirow[t]{2}{*}{ Sample } & \multicolumn{3}{|c|}{ Melting } & \multicolumn{3}{|c|}{ Freezing } \\
\hline & $\mathrm{T}_{\text {onset }}{ }^{\mathrm{a}}\left({ }^{\circ} \mathrm{C}\right)$ & $\mathrm{T}_{\text {Peak }}{ }^{\mathrm{b}}\left({ }^{\circ} \mathrm{C}\right)$ & $\begin{array}{c}\text { Latent } \\
\text { heat } \\
(\mathrm{J} / \mathrm{g})\end{array}$ & $\mathrm{T}_{\text {onset }}\left({ }^{\circ} \mathrm{C}\right)$ & $\mathrm{T}_{\text {Peak }}\left({ }^{\circ} \mathrm{C}\right)$ & $\begin{array}{c}\text { Latent } \\
\text { heat } \\
(\mathrm{J} / \mathrm{g})\end{array}$ \\
\hline$n$-octadecane & 25.1 & 32.9 & 225.4 & 25.1 & 18.5 & 225.2 \\
\hline MicroPCMs & 24.5 & 30.1 & 90.6 & 25.2 & 18.7 & 90.0 \\
\hline PPy/MicroPCMs & 24.6 & 30.0 & 90.2 & 24.8 & 17.9 & 92.0 \\
\hline
\end{tabular}

${ }^{\mathrm{a}} \mathrm{T}_{\text {onset }}$ - onset temperature on DSC curve; ${ }^{\mathrm{b}} \mathrm{T}_{\text {Peak }}$ - peak temperature on DSC curve.

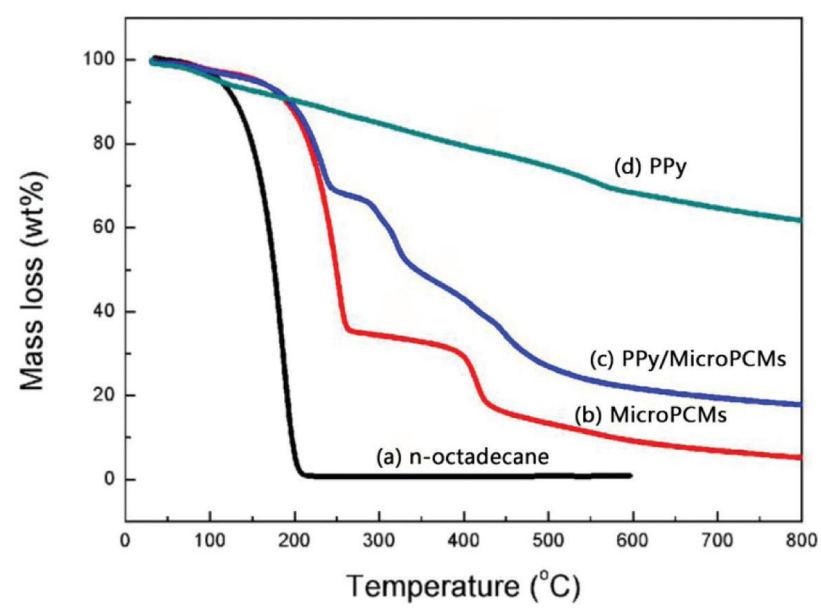

Figure 6: TG curves of (a) $n$-octadecane, (b) MicroPCMs, (c) PPy/MicroPCMs, (d) PPy.

or thickness of the shell layer. The conductivity of the composites increases from $0.1 \mathrm{~S} \cdot \mathrm{cm}^{-1}$ to $0.33 \mathrm{~S} \cdot \mathrm{cm}^{-1}$ as the PPy concentration increases from 3 to $10 \mathrm{wt} \%$. In contrast, the electrical conductivity of PPy is $0.5 \mathrm{~S} \cdot \mathrm{cm}^{-1}$. Obviously, the conductivity of these composites is lower than that of pure PPy, since the thickness of PPy on MicroPCMs is thinner.

\section{Conclusions}

Microencapsulated phase change materials were fabricated using $n$-octadecane as PCM, and melamine-formaldehyde as shell, via the in situ polymerization technique. The microcapsules were coated with polypyrrole to fabricate conductive microcapsules. The fabricated microcapsules have a homogeneous spherical profile and core/shell structure. The melting and freezing latent heats of the composites are 90.2 and $92.0 \mathrm{~J} / \mathrm{g}$, respectively, while the mass percentage of the $n$-octadecane in the composites is

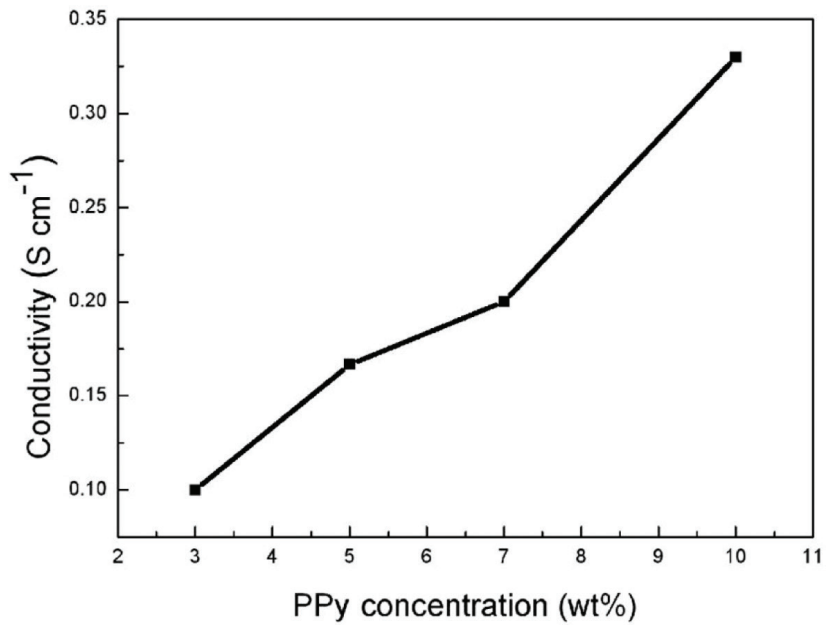

Figure 7: Effects of PPy contents in fabrication of PPy/MicroPCMs on conductivity.

49.7\%. The thermal stability of heat-treated PPy/MicroPCMs was enhanced. The PPy/MicroPCMs exhibit a fairly high electrical conductivity of about $0.33 \mathrm{~S} \cdot \mathrm{cm}^{-1}$ by adjusting the content of PPy. The electrical conductivity and phase change properties of PPy/MicroPCMs may be used in the field of camouflage technology and electronic cooling. The phase change material microcapsules can be used in the textile field to prepare fibers and fabrics with the functions of antistatic, heat storage and temperature regulation.

\section{References}

1. Jamekhorshid A., Sadrameli S.M., Farid M., A review of microencapsulation methods of phase change materials (PCMs) as a thermal energy storage (TES) medium. Renew. Sust. Energ. Rev., 2014, 31, 531-542.

2. Li M., Wu Z.S., Kao H.G., Study on preparation and thermal properties of binary fatty acid/diatomite shape-stabilized phase change materials. Sol. Energ. Mat. Sol. C., 2011, 95, 2412-2416. 
3. Farid M.M., Khudhair A.M., Razack S.A.K., Al-Hallaj S., A review on Phase Change Energy Storage: Materials and Applications. Energ. Convers. Manage., 2004, 45, 1597-1615.

4. Khudhair A.M., Farid M.M., A review on energy conservation in building applications with thermal storage by latent heat using phase change materials. Energ. Convers. Manage., 2004, 45, 263-275.

5. Das S., Dutta T.K., Mathematical modeling and experimental studies on solar energy storage in a phase change material. Sol. Energy, 1993, 51, 305-312.

6. Enibe S.O., Thermal analysis of a natural circulation solar air heater with phase change material energy storage. Renew. Energ., 2003, 28, 2269-2299.

7. Kenisarin M., Mahkamov K., Solar energy storage using phase change materials. Renew. Sust. Energ. Rev., 2007, 11, 1913-1965.

8. Varol Y., Koca A., Oztop H.F., Avci E., Forecasting of thermal energy storage performance of phase change material in a solar collector using soft computing techniques. Expert. Syst. Appl., 2010, 37, 2724-2732.

9. Jegadheeswaran S., Pohekar S.D., Kousksou T., Investigations on thermal storage systems containing micron-sized conducting particles dispersed in a phase change material. Energy Proced., 2012, 14, 643-648.

10. Zhang X.X., Wang X.C., Tao X.M., Yick K.L., Energy storage polymer/MicroPCMs blended chips and thermo-regulated fibers. J. Mater. Sci., 2005, 40, 3729-3734.

11. Li H., Fang G.Y., Liu X., Synthesis of shape-stabilized paraffin/ silicon dioxide composites as phase change material for thermal energy storage. J. Mater. Sci., 2010, 45, 1672-1676.

12. Zhang X.X., Fan Y.F., Tao X.M., Yick K.L., Fabrication and properties of microcapsules and nanocapsules containing $n$-octadecane. Mater. Chem. Phys., 2004, 88, 300-307.

13. Fan Y.F., Zhang X.X., Wu S.Z., Wang X.C., Thermal stability and permeability of microencapsulated $n$-octadecane and cyclohexane. Thermochim. Acta, 2005, 429, 25-29.

14. You M., Zhang X.X., Li W., Wang X.C., Effects of MicroPCMs on the fabrication of MicroPCMs/ polyurethane composite foams. Thermochim. Acta, 2008, 472, 20-24.
15. You M., Zhang X.X., Wang X.C., Zhang L., Wen W., Effects of type and contents of microencapsuled $n$-alkanes on properties of soft polyurethane foams. Thermochim. Acta, 2010, 500, 69-75.

16. Jiang M.J., Song X.Q., Xu J.J., Ye G.G., Preparation of a new thermal regulating fiber based on PVA and paraffin. Sol. Energ. Mat. Sol. C., 2008, 92, 1657-1660.

17. Salaün F., Devaux E., Bourbigot S., Rumeau P., Thermoregulating response of cotton fabric containing microencapsulated phase change materials. Thermochim. Acta, 2010, 506, 82-93.

18. Wang L., Meng D., Fatty addeutetic-polymethylmethac-rylate composite as form-stable phase change material for thermal energy storage. Appl. Energ., 2010, 87, 2660-2665.

19. Karaipekli A., Sarı A., Capric-myristic acid/expanded perlite composite as form-stable phase change material for latent heat thermal energy storage. Renew. Energ., 2008, 33, 2599-2605.

20. Wang Y., Xia T.D., Zheng H., Feng H.X., Stearic acid/silica fume composite as form-stable phase change material for thermal energy storage. Energ. Buildings, 2011, 43, 2365-2370.

21. Chen C., Wang L., Huang Y., Electrospun phase change fibers based on polyethylene glycol/cellulose acetate blends. Appl. Energ., 2011, 88, 3133-3139.

22. Ju C.B., Wang Y.S., He D.W., Gao Q., Gao L., Fu M., Synthesis and Infrared Property of Polyaniline/Phase-Change Nanocapsule Composite. J. Nanosci. Nanotechno., 2011, 11, 9665-9670.

23. Songa K.T., Lee J.Y., Kim H.D., Kim D.Y., Kim S.Y., Kim C.Y., Solvent effects on the characteristics of soluble polypyrrole. Synthetic Met., 2000, 110, 57-63.

24. Yang C., Liu P., Wang T., Well-defined core-shell carbon black/ polypyrrole nanocomposites for electrochemical energy storage. ACS Appl. Mater. Inter., 2011, 3(4), 1109-1114.

25. Chao Y., Peng L., Ting M.W., Well-Defined Core-Shell Carbon Black/Polypyrrole nanocomposites for electrochemical energy storage. ACS Appl. Mater. Inter., 2011, 3(4), 1109-1114.

26. Müllera D., Rambo C.R., Recouvreux D.O.S., Porto L.M., Barra G.M.O., chemical in situ polymerization of polypyrrole on bacterlal cellulose nanofibers. Synthetic Met., 2011, 161, 106-111. 